\title{
Malformaciones congénitas en Chile y Latino América: Una visión epidemiológica del ECLAMC del período 1995-2008
}

\author{
JULIO NAZER $\mathrm{H}^{1}$., LUCÍA CIFUENTES O. ${ }^{2}$
}

'Unidad de Neonatología, Maternidad Hospital Clínico Universidad de Chile.

${ }^{2}$ Instituto de Ciencias Biomédicas, Facultad de Medicina, Universidad de Chile. Santiago de Chile.

Recibido el 21 de julio de 2010, aceptado el 9 de diciembre de 2010.

Correspondencia a: Dr. Julio Nazer $\mathrm{H}$. Santos Dumont 999, Santiago. Chile. E-mail: jnazer@ redclinicauchile.cl

\section{Congenital malformations in Latin America in the period 1995-2008}

Background: The Latin American Study of Congenital Malformations (ECLA$M C$ ) has performed an epidemiological surveillance of congenital malformations since 1967. This allows to detect any unexpected change in the incidence of malformations, possibly caused by a new environmental teratogenic agent. Aim: To report a summary of the results thus far obtained in this study. Material and Methods: The ECLAMC database was analyzed and all live births and stillbirths of more than 500 grams in the period 1995-2008, were analyzed. Results: There were 2,409,407 births in the nine participant countries. Of these 31,516 (1.3\%) were stillbirths. The global rate of congenital malformations in this sample was $2.7 \%$. In the studied period, there was a significant reduction in the rates of anencephaly and spina bifida in Chile and Argentina. In the rest of the countries, the global rates of malformations increased. Venezuela had the higher rate of teenage pregnancies (25\%), followed by Colombia (23\%). Chile had the higher percentage of women aged 35 years or more giving birth (14\%), followed by Uruguay (13\%). However, Chile had the higher rate of Down syndrome and Uruguay, the lowest (24.7 and 13.6 per 10000). Conclusions: There is a tendency towards an increase in the rates of congenital malformations in this sample, with significant differences among countries.

(Rev Med Chile 2011; 139: 72-78).

Key words: Down syndrome; Nervous system malformations; Stillbirth.
$\mathrm{E}$ 1 ECLAMC (Estudio Colaborativo Latino Americano de Malformaciones Congénitas) fue creado por el Profesor Dr. Eduardo Castilla "como un programa de investigación clínica y epidemiológica de los factores de riesgo de la etiología de las anomalías congénitas en hospitales Latino Americanos usando una metodología caso-control".

Comenzó a operar en 1967 como una investigación limitada a la ciudad de Buenos Aires, Argentina, y gradualmente se expandió hasta integrar a 10 países de América del Sur. Desde 1974 el ECLAMC ha sido miembro fundador del International Clearinghouse for Birth Defects Mo- nitoring Systems que reúne a más de 40 Programas de otras regiones del mundo. Es Centro colaborador de la Organización Mundial de la Salud para la prevención de malformaciones congénitas y desde el año 2000 es un miembro colaborador de la Red Global para la investigación en la salud de las mujeres y niños del NIH. Todos los Programas que participan lo hacen con el mismo sistema, por lo que sus resultados son comparables. La red de maternidades del ECLAMC examina e incorpora alrededor de 200.000 nacimientos al año y tiene hasta el momento más de 5 millones de nacimientos con más de 300.000 casos y otros tantos controles. 
La función principal del ECLAMC es hacer vigilancia epidemiológica de las malformaciones congénitas con el fin de pesquisar cualquier aumento brusco de la frecuencia de alguna malformación que podría estar indicando la presencia de algún teratógeno nuevo.

Es fundamental también dar a conocer periódicamente los resultados con el fin que la comunidad médica de cada país y también de la región esté informada.

\section{Pacientes y Método}

Se registran todos los recién nacidos (RN) portadores de una o más malformaciones mayores y menores que pesen 500 gramos o más, vivos o mortinatos, de acuerdo a un Manual Operacional $^{1}$. Se selecciona como control para cada caso al RN, del mismo sexo, nacido a continuación, en el mismo establecimiento y que no presente malformaciones.

Para este estudio se considera el total de nacimientos en cada uno de los países participantes $y$ en el total del ECLAMC en el período 19952008. Estos países son: Argentina, Bolivia, Brasil, Colombia, Chile, Ecuador, Paraguay, Uruguay y Venezuela.

El objetivo de este trabajo es dar a conocer lo que está ocurriendo en Latinoamérica en lo que se refiere a prevalencia al nacimiento de malfor- maciones congénitas; compararlas entre los países participantes y con lo comunicado anteriormente por nosotros ${ }^{2}$; estudiar la distribución etaria de las madres de los distintos países con el fin de conocer la proporción de madres adolescentes y mayores y relacionarlas con las prevalencias de distintas anomalías; conocer la Mortalidad fetal tardía o Mortinatalidad, global y la de cada país.

\section{Resultados}

En el período estudiado ocurrieron 2.409.407 nacimientos, 2.377.891 nacidos vivos (NV) y 31.516 mortinatos (NM) (Tabla 1). Esto representa una Mortinatalidad Global de 1,3\%. Este rubro es muy heterogéneo en los distintos países participantes, desde $0,7 \%$ en Chile a 2,2\% en Bolivia $\left(\chi^{2}=2.245 ; \mathrm{p}<0,0001\right)$; siendo Argentina, Uruguay y Chile los países que presentan tasas de mortinatalidad significativamente inferiores al promedio latinoamericano (Tabla 1).

La tasa de prevalencia al nacimiento de malformaciones congénitas en los 9 países participantes es también muy diferente entre todos los países $\left(\chi^{2}=6.744,0 ; \mathrm{p}<0,0001\right)$.

La Tasa global de malformaciones en el total del ECLAMC es de 2,7\% con una dispersión que va desde $1,4 \%$ en Ecuador hasta 4,2\% en Brasil $(\mathrm{p}<0,00001)$. Este último y Chile son los países que ostentan tasas superiores al promedio latino-

Tabla 1. Distribución por país del total de nacimientos, nacidos vivos, mortinatos, total de malformados, malformados vivos, malformados mortinatos. Tasas por 100

\begin{tabular}{|lrrrrrrrrrr|}
\hline País & $\begin{array}{c}\text { Total } \\
\text { nac }\end{array}$ & NV & NM & \% & $\begin{array}{c}\text { Total } \\
\text { MF }\end{array}$ & \% & MFV & \% & MFM & \% \\
Argentina & 815.454 & 805.455 & 9.999 & $1,2^{*}$ & 17.995 & $2,2^{*}$ & 17.344 & 2,2 & 651 & 6,5 \\
Bolivia & 107.284 & 104.917 & 2.367 & $2,2^{*}$ & 1.902 & $1,8^{*}$ & 1.756 & 1,7 & 146 & 6,2 \\
\hline Brasil & 517.842 & 510.402 & 7.440 & $1,4^{*}$ & 21.557 & $4,2^{* *}$ & 20.455 & 4,0 & 1.102 & $14,8^{*}$ \\
\hline Colombia & 68.150 & 66.989 & 1.161 & $1,7^{*}$ & 1.290 & $1,9^{*}$ & 1.290 & 1,8 & 120 & 10,3 \\
\hline Chile & 374.640 & 371.914 & 2.726 & $0,7^{*}$ & 11.666 & $3,1^{*}$ & 11.350 & 3,1 & 316 & 11,6 \\
\hline Ecuador & 136.147 & 134.399 & 1.748 & $1,3^{*}$ & 1.852 & $1,4^{* *}$ & 1.812 & 1,4 & 40 & 2,3 \\
\hline Paraguay & 26.805 & 26.331 & 474 & $1,8^{*}$ & 581 & $2,2^{*}$ & 566 & 2,2 & 15 & $3,2^{*}$ \\
\hline Uruguay & 94.499 & 93.462 & 1.037 & $1,1^{*}$ & 2.302 & $2,4^{*}$ & 2.223 & 2,4 & 79 & 7,6 \\
\hline Venezuela & 236.193 & 232.028 & 4.165 & $1,8^{*}$ & 5.559 & $2,4^{*}$ & 5.359 & 2,3 & 200 & 4,8 \\
Total ECLAMC & 2.409 .407 & 2.377 .891 & 31.516 & $1,3^{*}$ & 65.581 & $2,7^{*}$ & 62.865 & 2,6 & 2.716 & 8,6 \\
\hline
\end{tabular}

${ }^{*} p<0,0001 ;{ }^{* *} p<0,00001$. 
岂

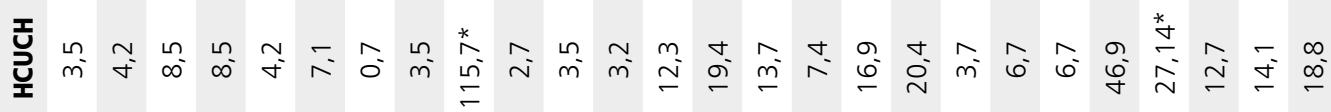

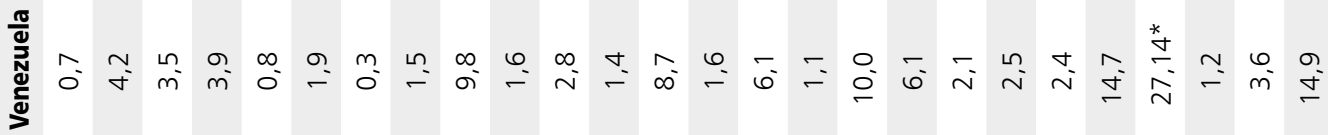

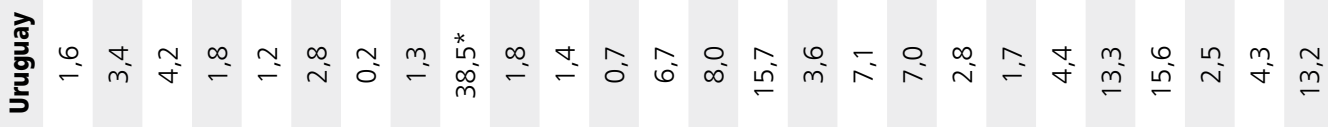

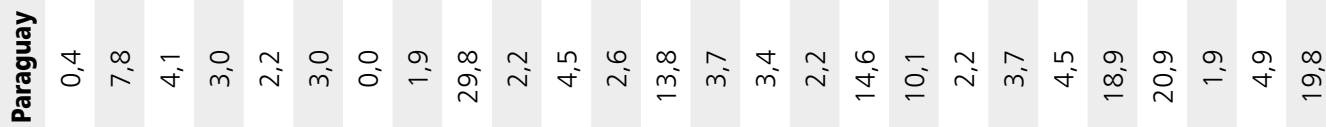
旁

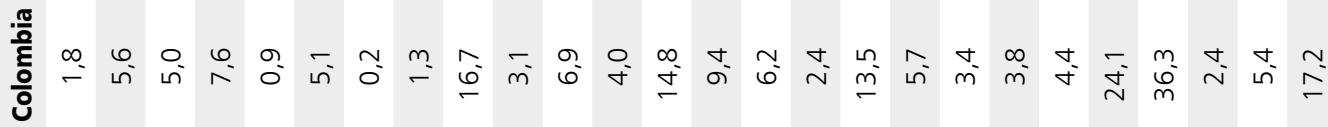

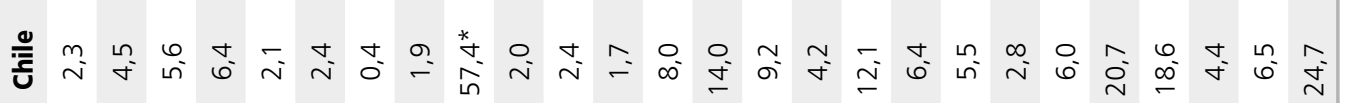

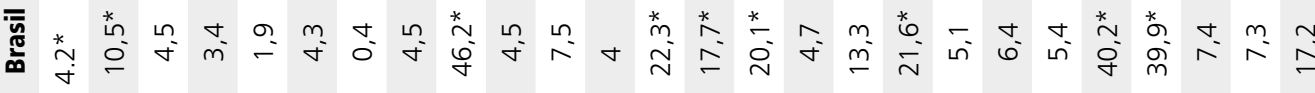
㟦 产

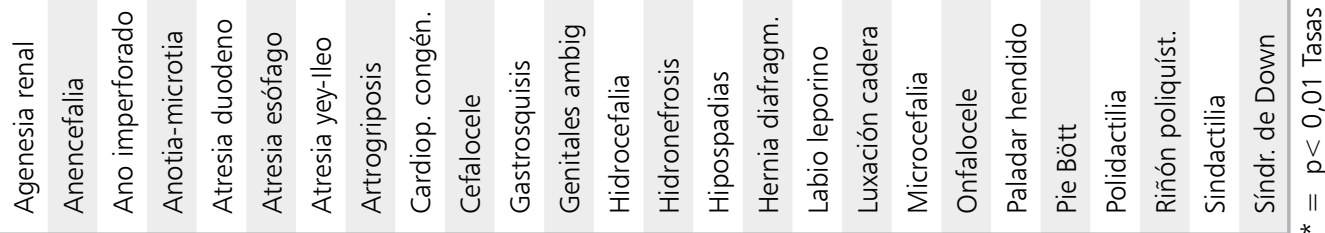


americano. Todo el resto de los países presentan tasas significativamente inferiores al promedio $(\mathrm{p}<0,001)$ (Tabla 1).

Esta dispersión o heterogeneidad se ve tanto en NV como en NM, especialmente entre estos últimos, donde Paraguay $(3,2 \%)$ presenta la menor tasa y Brasil $(14,8 \%)$ la mayor. Esta diferencia en NM se podría explicar por la falta de autopsias en los países con frecuencias bajas.

Para comprender estas diferencias se comparó las frecuencias de 26 malformaciones congénitas de diagnóstico confiable entre los diferentes países y que se presentan en la Tabla 2. En ella se aprecia que existen diferencias significativas en los diferentes países para todos los diagnósticos analizados $(p<0,001)$. En términos generales Brasil presenta las tasas más altas en casi todos los casos (excepto anotia-microtia y ano imperforado) y Bolivia con Uruguay presentan tasas más bajas que el resto en numerosos diagnósticos. La Tasa global de malformaciones congénitas del total del ECLAMC fue de 18,8 por 10.000 nacimientos.

Si comparamos lo que ha ocurrido con la evolución de las tasas de prevalencia de algunas malformaciones congénitas entre Chile y el ECLAMC, en dos períodos 1982-1994 y 1995-2005 (Tabla 3), podemos apreciar que en la mayoría de los diagnósticos las tasas han aumentado, tanto en Chile como en el total del ECLAMC, en forma significativa, como anotia-microtia, hidronefrosis, hipospadias, microcefalia y especialmente síndrome de Down $(p<0,05)$. Sin embargo, en Chile disminuyeron significativamente las tasas de anencefalia de 7,7 por 10.000 en 1982-94 a 4,5

Tabla 3. Comparación de tasas de MF entre total de Chile y total del ECLAMC. Tasas por 10.000

\begin{tabular}{|c|c|c|c|c|}
\hline \multirow{2}{*}{$\begin{array}{l}\text { Período } \\
\text { Diagnósticos }\end{array}$} & \multicolumn{2}{|c|}{ 1982-1994 } & \multicolumn{2}{|c|}{ 1995-2008 } \\
\hline & Chile & ECLAMC & Chile & ECLAMC \\
\hline Agenesia renal & 2,06 & 1,1 & 2,3 & $2,4^{* *}$ \\
\hline Anencefalia & 7,7 & 6,8 & $4,5^{*}$ & 6,6 \\
\hline Ano imperforado & 4,9 & 4,0 & 5,6 & $5,3^{* *}$ \\
\hline Anotia-microtia & 4,7 & 4,2 & $6,4^{*}$ & $5,4^{* *}$ \\
\hline Atresia de duodeno & 1,1 & 0,9 & $2,1^{*}$ & $1,5^{* *}$ \\
\hline Atresia de esófago & 3,5 & 2,7 & $2,4^{*}$ & $3,5^{* *}$ \\
\hline Atresia de yeyuno & 0,7 & 0,6 & 0,4 & $0,4^{* *}$ \\
\hline Cefalocele & 2,0 & 1,8 & 2,0 & $2,1 * *$ \\
\hline Espina bífida & 10,8 & 7,1 & $5,7^{*}$ & $8.2^{* *}$ \\
\hline Genitales ambiguos & 2,3 & 2,0 & 1,7 & 2,2 \\
\hline Hernia diafragmática & 3,9 & 1,8 & 4,2 & $3,6^{* *}$ \\
\hline Hidrocefalia & 5,9 & 6,2 & $8,0^{*}$ & $12,3^{* *}$ \\
\hline Hidronefrosis & 2,7 & 2,1 & $14,0 *$ & $8,7^{* *}$ \\
\hline Hipospadias & 6,8 & 8,1 & $9,2^{*}$ & $9,5^{* *}$ \\
\hline Labio leporino & 12,0 & 10,6 & 12,1 & $13,3^{* *}$ \\
\hline Microcefalia & 2,7 & 2,6 & $5,5^{*}$ & $3,7^{* *}$ \\
\hline Onfalocele & 2,6 & 2,3 & 2,8 & $3,6^{* *}$ \\
\hline Paladar hendido & 4,3 & 3,6 & $6,0^{*}$ & $4,6^{* *}$ \\
\hline Riñón poliquístico & 3,3 & 1,7 & $4,4^{*}$ & $3,9 * *$ \\
\hline Síndrome de Down & 17,3 & 15,3 & $24,7^{*}$ & $18,8^{* *}$ \\
\hline Total nacimientos & 241.505 & 2.446 .663 & 374.640 & 2.409 .407 \\
\hline
\end{tabular}

${ }^{*} p<0,05$ Chile antes y después; ${ }^{* *} p<0,05$ ECALMC antes y después. 
por 10.000 en el $2005(p<0,05)$. Lo mismo espina bífida, que bajó de 10,8 por 10.000 a 5,7 por 10.000 , y atresia de esófago que bajó de 3,5 a 2,4 por $10.000(\mathrm{p}<0,05)$.

Se estudiaron varios factores de riesgo para tener hijos con malformaciones congénitas, entre los que destaca por su importancia reconocida la mayor edad materna, por lo que decidimos comparar la estructura etárea materna entre los distintos países separando a las madres en tres grupos: 19 años y menos, 35 años y más y de 20 a 34 años (Tabla 4). Las más altas frecuencias de embarazo en adolescentes ocurren en Venezuela, Brasil, Colombia y Paraguay. El mayor porcentaje de madres mayores de 34 años ocurre en Chile y Uruguay.

Al estudiar la frecuencia de nacimientos según grupos etarios maternos en los distintos países, llama la atención las diferencias que hay entre ellos, por ejemplo en Venezuela la cuarta parte de los nacimientos corresponde al grupo de madres adolescentes, 20 años o menos (25,3\%), le sigue Colombia con $23,3 \%$ y Paraguay con $21,7 \%$. La cifra promedio de todo el ECLAMC es de 19,7\%. Bajo ese promedio están Uruguay con 15,6\%, Chile con $17,5 \%$ y Bolivia con $18,1 \%$ (Tabla 4 ).

El promedio de todo el ECLAMC para el grupo de madres de 35 años y más es $11,3 \%$ y están por sobre él Chile con el porcentaje mayor $14,4 \%$, seguido por Uruguay 13,6\% y Bolivia y Paraguay con
$12 \%$; mientras que Colombia con $6,3 \%$ seguido de Venezuela son los países con menor proporción de madres mayores de 34 años.

Chile y Uruguay representan una situación especial: tienen las frecuencias más bajas de nacimientos de madres adolescentes y las más altas en mujeres mayores de 34 años. Sin embargo, lo que ocurre en estos dos países con síndrome de Down, que es la patología en la que más incidiría la mayor edad materna es interesante de estudiar, ya que en Chile se cumple lo esperado, que por presentar el mayor porcentaje de madres de mayor edad, tiene la mayor frecuencia de síndrome de Down, 24,7 por 10.000 , pero Uruguay que tiene la tasa más baja de síndrome de Down de Latinoamérica (13,2 por 10.000) posee la segunda mayor proporción de madres mayores. Esto podría significar que edad materna es un factor de riesgo importantísimo para esta patología, pero parece no ser el único factor. Habría que estudiar otros factores como etnicidad, por ejemplo. Se ha comunicado que las madres de origen hispánico tienen mayor riesgo de tener hijos con síndrome de Down ${ }^{3,4}$. La composición étnica de Chile tiene influencia hispánica e indoamericana, Uruguay tiene componentes europeos y africanos.

Si estudiamos que está ocurriendo con la evolución de las tasas de prevalencia al nacimiento de las MFC en Chile y Latino América encontramos que en Chile, por ejemplo, la tasa global para

Tabla 4. Distribución de los nacimientos según grupos etarios de sus madres, por país y total del ECLAMC

\begin{tabular}{|lrrrrrrr|}
\hline $\begin{array}{l}\text { Grupos de } \\
\text { edades }\end{array}$ & $<=\mathbf{1 9}$ años & $\mathbf{\%}$ & $\mathbf{2 0} \mathbf{a} \mathbf{3 4}$ años & $\mathbf{\%}$ & $\boldsymbol{>}=\mathbf{3 5}$ años & $\mathbf{\%}$ & Total \\
Argentina & 156.856 & 19,2 & 568.721 & 69,7 & 89.877 & 11,0 & 815.454 \\
Bolivia & 19.387 & 18,1 & 75.011 & 69,1 & 12.886 & 12,0 & 107.284 \\
Brasil & 10.4946 & 20,3 & 356.295 & 68,0 & 56.601 & 10,9 & 517.842 \\
Colombia & 15.902 & 23,3 & 44.987 & 66,0 & 7.261 & 6,3 & 68.150 \\
Chile & 65.565 & 17,5 & 255.233 & 68,1 & 53.842 & 14,4 & 374.640 \\
Ecuador & 27.302 & 20,0 & 94.897 & 69,7 & 13.948 & 10,2 & 136.147 \\
Paraguay & 5.820 & 21,7 & 17.779 & 63,3 & 3.206 & 12,0 & 26.805 \\
Uruguay & 14.688 & 15,6 & 67.034 & 71,0 & 12.777 & 13,6 & 94.449 \\
Venezuela & 59.652 & 25,3 & 158.032 & 67,0 & 18.509 & 7,8 & 236.193 \\
Total ECLAMC & 475.711 & 19,7 & 1.660 .620 & 68,9 & 273.076 & 11,3 & 2.409 .407 \\
\hline
\end{tabular}

$\mathrm{p}<0,001$. 
el período 1982-88 era de 2,97\%; en el período 1989-94 fue de 2,6\%; en 1995-99 subió a 3,96\%; en 2001-03 bajó a 2,3\% para volver a subir en el período 3,1\% en 1998-2005. Es decir, que existe una tendencia a aumentar las tasas de prevalencia de malformaciones congénitas.

\section{Discusión}

La Mortalidad fetal tardía o Mortinatalidad es muy heterogénea en los distintos países participantes, desde $0,7 \%$ en Chile a 2,2\% en Bolivia. $\left(\chi^{2}=2.245 ; \mathrm{p}<0,0001\right)$; siendo Argentina y Chile los países que presentan tasas de mortinatalidad significativamente inferiores al promedio latinoamericano. Esta dispersión se podría explicar por las diferencias que puede haber en la calidad de la atención prenatal y controles del embarazo.

La gran heterogeneidad de las tasas de prevalencia al nacimiento de las malformaciones congénitas en los distintos países latinoamericanos participantes del ECLAMC es un hecho que es necesario tratar de explicar. Sabemos que hay factores ambientales, genéticos y étnicos, además de la edad materna, que pueden dar alguna luz al respecto y que son muy diferentes en los países sudamericanos, como lo vimos en el párrafo anterior. Es sabido que gastrosquisis se presenta con mayor frecuencia en hijos de madres adolescentes ${ }^{5-7}$; pues bien, esta anomalía se presentó con significativa mayor frecuencia en Brasil y Colombia, países que tienen la mayor proporción de madres de 20 años y menores de esa edad. Chile tiene la tasa más alta de síndrome de Down (24,7 por 10.000 ) y es el país con el porcentaje más alto de mujeres de 35 años y más. Se conoce el hecho que anotia-microtia se presenta más frecuentemente en ciudades de gran altura sobre el nivel del mar como en Ecuador y Bolivia ${ }^{8,9}$. Labio leporino se asocia con origen étnico amerindio lo que se correlaciona con las altas tasas de esas malformaciones en Bolivia y Ecuador $^{10}$, mientras que polidactilia se asocia con origen étnico africano lo que está refrendado al ver las altas tasas en Brasil, Colombia y Ecuador ${ }^{11-13}$.

Pero si analizamos malformación por malformación nos encontramos que hay algunas que han bajado significativamente, como los defectos de cierre del tubo neural, espina bífida, anencefalia y cefalocele. Hay un hecho muy importante que ha estado influenciando en algunos países la frecuencia de estas malformaciones y que es la fortificación de la harina con ácido fólico con el fin de hacer prevención primaria de esas anomalías. En efecto, las tasas de defectos de cierre del tubo neural disminuyeron en forma significativa $(\mathrm{p}<$ 0,02) en Chile, Argentina. En Brasil, espina bífida disminuyó, pero no alcanzó a ser significativo, seguramente porque al momento de procesar estos datos sólo llevaba menos de dos años iniciado el Programa (Tabla 5). Chile comenzó a fortificar la harina en el año 2001 y al 2007 se había logrado

Tabla 5. Tasas de prevalencia al nacimiento de defectos del tubo neural (aislados y total) en los períodos pre y post fortificación de la harina con ácido fólico en tres países sudamericanos, según datos del ECLAMC

\begin{tabular}{|c|c|c|c|c|c|c|c|c|c|c|c|c|}
\hline \multirow[t]{3}{*}{ Diagnósticos } & \multicolumn{4}{|c|}{ Chile } & \multicolumn{4}{|c|}{ Argentina } & \multicolumn{4}{|c|}{ Brasil } \\
\hline & \multicolumn{2}{|c|}{ Pre fortif. } & \multicolumn{2}{|c|}{ Post-fortif. } & \multicolumn{2}{|c|}{ Pre fortif. } & \multicolumn{2}{|c|}{ Post-fortif. } & \multicolumn{2}{|c|}{ Pre fortif. } & \multicolumn{2}{|c|}{ Post-fortif. } \\
\hline & Aislado & Total & Aislado & Total & Aislado & Total & Aislado & Total & Aislado & Total & Aislado & Total \\
\hline Anencefalia & 0,52 & 0,63 & 0,26 & $0,37 *$ & 0,69 & 0,86 & 0,29 & $0,37^{*}$ & 0,90 & 1,12 & 0,45 & 0,69 * \\
\hline Espina bífida & 0,73 & 1,02 & 0,24 & $0,46^{*}$ & 0,82 & 1,27 & 0,33 & $0,66^{*}$ & 0,86 & 1,45 & 0,69 & 1,42 \\
\hline Cefalocele & 0,26 & 0,33 & 0,09 & $0,18^{*}$ & 0,21 & 0,32 & 0,10 & 0,20 * & 0,31 & 0,57 & 0,12 & $0,32 *$ \\
\hline \multicolumn{13}{|l|}{$p<0,02$} \\
\hline Período & \multicolumn{2}{|c|}{$1998-2000$} & \multicolumn{2}{|c|}{ 2001-2003 } & 2002-2004 & \multicolumn{2}{|c|}{ 2005-2007 } & \multicolumn{3}{|c|}{ 2003-06.2005 } & \multicolumn{2}{|c|}{ 07.2005-2007 } \\
\hline $\begin{array}{l}\text { Nacimientos } \\
\text { observados }\end{array}$ & \multicolumn{2}{|c|}{69.677} & \multicolumn{2}{|c|}{243.624} & 193.509 & \multicolumn{2}{|c|}{147.853} & \multicolumn{3}{|c|}{102.751} & \multicolumn{2}{|c|}{92.843} \\
\hline $\begin{array}{l}\text { Nacimientos } \\
\text { por año }\end{array}$ & \multicolumn{4}{|c|}{244.000} & \multicolumn{3}{|c|}{685.000} & \multicolumn{5}{|c|}{3.000 .000} \\
\hline
\end{tabular}


una disminución global significativa $(\mathrm{p}<0,02)$ de cerca de $60 \% \%^{14,15}$. Espina bífida disminuyó su frecuencia en $54 \%$, cefalocele $47 \%$ y anencefalia $29 \%$. En Argentina ocurrió algo parecido: comenzó a fortificar la harina de trigo en el año 2004 y ha logrado una reducción global de $44 \%$. Espina bífida 50,3\%, anencefalia 59,5\%. Brasil comenzó a fortificar la harina a fines de 2005 y ha logrado una disminución (no significativa) de $23,5 \%$ de reducción en espina bífida y $54,5 \%$ de reducción significativa de anencefalia, según datos entregados por el ECLAMC (Tabla 5).

Agradecimientos: Agradecemos sinceramente a la Coordinación Central del ECLAMC en las personas del Dr. Eduardo Castilla y del Dr. Jorge López-Camelo por habernos permitido el acceso a su base de datos, sin lo que no habría sido posible realizar este trabajo.

\section{Referencias}

1. Manual Operacional. ECLAMC. Edición 2002. Ed Fiocruz. Río de Janeiro. Brasil.

2. Nazer J, Cifuentes L, Meza M. Incidencia de las malformaciones congénitas en 10 maternidades chilenas participantes del ECLAMC. Rev Med Chile 1997; 125: 993-1001.

3. Carothers AD, Hectch CA, Hook EB. International variation in reported livebirth prevalence rates of Down Sindrome, adjusted for maternal age. J Med Genet 36; 386-93.

4. Canfield MA, Honein MA, Yuskiv N, Xing J, Mai CT, Collins JS, et al. National stimates and race/ethnic-specific variation of selected birth defects in the United States, 1999-2001. Birth defects Res A Clin Mol Teratol 2006; 76: 747-56.
5. Nazer J, Cifuentes L, Aguila A,Ureta P, Bello MP, Correa $\mathrm{F}$, et al. Edad materna y malformaciones congénitas. Un registro de 35 años. 1970-2005. Rev Med Chile 2007; 135: 1463-69.

6. Pardo RA, Nazer J, Cifuentes L. Incidencia de defectos congénitos y bajo peso de nacimiento en madres adolescentes. Rev Med Chile 2003; 131: 1165-72.

7. Pennan DG, Fisher RM, Noblett HR. Increase in incidence of gastroschisis in the South West of England in 1995. Br J Obstet Gynecol 1998; 105: 228-32.

8. Nazer J, Lay-Son R, Cifuentes L. Prevalencia al nacimiento de Anotia-Microtia. Maternidad del Hospital Clínico de la Universidad de Chile. Período 1983-2005. Rev Med Chile 2006; 134: 1288-94.

9. Castilla EE, Orioli IM. The Latin American Collaborative Study of Congenital Malformation. Community Genetics 2004; 7: 76-94.

10. Castilla E, López-Camelo J, Paz J. Em Atlas Geográfico de lãs Malformaciones Congênitas em Sud América. Ed. Fiocruz. Rio de Janeiro. Brasil. 1995.

11. Erickson JD. Racial variations in the incidente of congenital malformations. Ann Hum Genet London 1976; 39: 315-20.

12. Castilla EE, Paz J, Mutchinick O. Polydactyly: Genetic study in South America. Am J Hum Genet 1973; 25: 405-12.

13. Cifuentes L, Nazer J. Polidactilia: Un estudio genéticoepidemiológico. Rev Med Chile 1996; 124: 313-18.

14. Castilla EE, Orioli IM, López-Camelo J, Dutra MG, Nazer J. Preliminary data of changes in Neural Tube Defects prevalence rates alter folic acid fortification in South America. Am J Med Genet 2003; 123A: 123-8.

15. Nazer J, Cifuentes L, Aguila A, Juárez ME, Cid MP, Godoy ML, et al. Efectos de la fortificación de la harina con ácido fólico sobre la evolución de las tasas de prevalencia al nacimiento de malformaciones congénitas en los hospitales chilenos del ECLAMC. Rev Med Chile 2007; 135: 198-204. 